\title{
Prostatic Pain
}

National Cancer Institute

\section{Source}

National Cancer Institute. Prostatic Pain. NCI Thesaurus. Code C78574.

Pain in the prostate gland. 